\title{
It all falls into place...
}

Researchers working on molecular self-assembly have never lacked ambition, but their dreams of producing commercially viable devices always looked like a distant goal. That may be about to change, says Philip Ball.

\begin{abstract}
magine a future in which computers build themselves. The nanoscale components of these machines would simply be brought together in solution and stirred gently. By tweaking the chemistry of the components so that some are attracted to each other, while others are repelled, the individual parts would assemble themselves, as if by magic, into a working whole.

Such is the hype generated by nanotechnology enthusiasts. But researchers working in this field have always regarded self-assembling computers as a distant dream. The chances of devising a commercially viable means for creating sophisticated electronic circuitry that builds itself remain remote.
\end{abstract}

But as the field evolves, more modest practical applications for selfassembly are emerging sooner than experts had dared hope. Some researchers are focusing on how to combine self-assembly with existing manufacturing methods. Others are developing new gene-screening techniques by using DNA to guide the self-assembly process. There has been little fanfare, but a smattering of start-up companies indicates that the ideas have commercial potential. "Self-assembly is going to be a big deal," predicts George Whitesides, a Harvard University chemist who has done some of the field's pioneering work.

\section{Causing a stir}

Much of the inspiration behind selfassembling systems comes from biology, where intricate molecular machines put themselves together without any external control. The ribosome, the site for protein synthesis in the cell, is a classic example. Human ribosomes are made up of about 80 separate proteins and four strands of ribonucleic acid. In common with many natural and artificial self-assembling systems, these components are held together by attractive forces which, compared with the bonds between the atoms within the components, are relatively easy to break.

Certain substances, such as detergents, can disrupt these forces, causing the ribosome to fall apart. But remove the detergent and the parts reassemble in the correct configuration, leaving a fully functioning ribosome. It is as if a dismantled watch could be rebuilt simply by throwing the parts together in a beaker of water and stirring.

The structures of the different components of the ribosome are key to this process. Each part is made up of a series of different groups of chemicals, and these interact with groups on other components in a well-defined way. The attractions and repulsions between these groups are such that the arrangement for a properly functioning ribosome is also the most stable one. Once recombined, the components naturally fall into this state.

The challenge for chemists working on self-assembly is to use their knowledge of molecular attraction and repulsion to devise artificial systems that will behave in the same way. And although mimicking the full glory of the ribosome remains a long-term goal, researchers have already created structures similar to simpler, repetitive biomolecular assemblies — such as the arrays of proteins that make up microtubules, part of the cellular skeleton, or the sheets of lipid molecules that line up to form cell membranes.

Whitesides and his Harvard colleagues are behind some of the

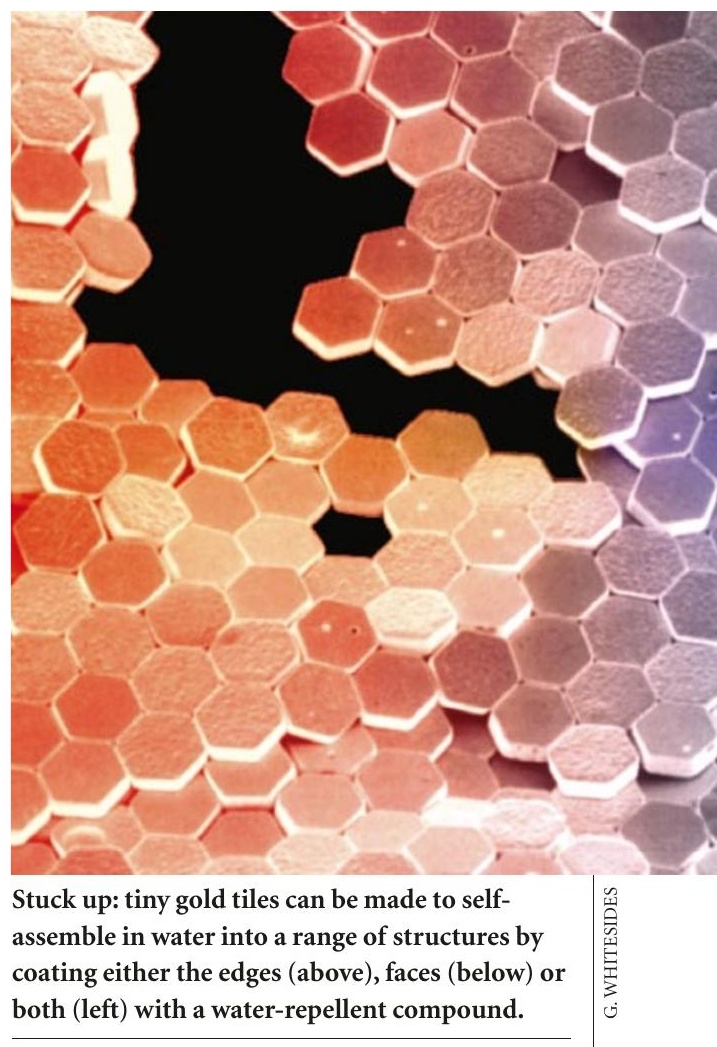

most distinctive work. They have created a range of repeating structures from simple units by coating particular surfaces of the units with substances that are either hydrophilic or hydrophobic (attracted to or repelled by water). When combined in water, the hydrophobic surfaces of the components tend to come together so as to minimize their contact with the surrounding liquid.

This July, Whitesides described how his team had applied this approach to flat hexagonal plates of gold just 50 nanometres thick and 10 micrometres wide ${ }^{1}$. Some parts of the plates were coated with chromium, which is hydrophilic. Others were coated with a film of hydrophobic molecules. The Harvard team mixed hundreds of thousands of the plates in water, and allowed them to selfassemble over a few days.

The structures that formed depended on where the coatings were applied. When the edges of the hexagons were made hydrophobic, the plates assembled into a sheet of hexagonal tiles. But if the hexagonal faces were made hydrophobic, the plates formed columns, like stacks of coins. If both faces and edges were coated, the result was a cluster of columns sticking side by side.

The hydrophobic forces are strong enough to hold the assemblies together, but not so strong that initial misalignments or partial overlaps of surfaces get fixed in place. 
Most of these irregularities are eventually corrected by the constant motion of the surrounding water and the plates themselves. By coating the hydrophobic surfaces with adhesives that set on exposure to either heat or light, the Harvard team managed to make the structures permanent.

The group has used a related technique to assemble log-shaped plastic components into miniature towers. Selected regions

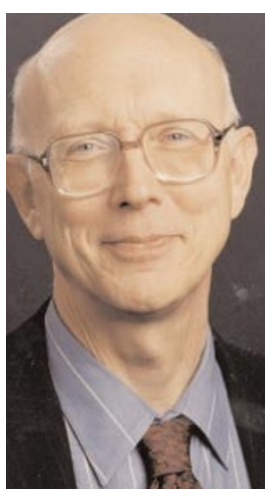

George Whitesides

has created 3D

electronic circuits. of the millimetresized logs were first coated with a bismuth alloy. The logs were then placed in water at a temperature above the alloy's melting point $-47^{\circ} \mathrm{C}$ - and gently stirred. As the logs came into contact with each other, areas of molten alloy stuck together. By applying the alloy to specific parts of the plastic components, Whitesides' team produced a system that selfassembled into a tower of criss-crossing $\log \mathrm{s}^{2}$.

Whitesides has also managed to create working electronic circuits out of millimetrescale plastic blocks. Some sides of the blocks had components, such as wires or lightemitting diodes, printed onto them. Others were designed so that they would join up with neighbouring blocks in specific ways. When gently agitated in water, the blocks spontaneously assembled into a working three-dimensional electronic circuit ${ }^{3}$.

Whitesides' circuits are still a long way from being a self-assembling computer, and the components needed a lot of careful hand-tooling, contrary to the ultimate aims of self-assembly. But his team has proved that working circuits can be self-assembled. If the researchers can repeat their work using blocks of micrometre dimensions, which are difficult to manipulate manually or robotically, they could point to way to building a new breed of ultra-small circuits.

\section{Building blocks}

In the short term, rather than using such techniques to create a completely new kind of manufacturing process, most researchers believe that self-assembly methods will be combined with standard chip-building technologies. This way, they argue, the convenience and low cost of self-assembly can be exploited where appropriate, leaving established methods to do the rest.

Last year, for example, Bob Wolkow and his colleagues at the Steacie Institute for Molecular Sciences in Ottawa, Canada, showed that styrene molecules will selfassemble into rows on a silicon surface that has been coated with hydrogen atoms ${ }^{4}$. The

\section{he emergence of new applications has happened sooner than expected.}

process is triggered by using a scanning tunnelling microscope to remove a single hydrogen atom from the surface, which creates a site at which a styrene molecule can bind. The binding process removes an adjacent hydrogen atom, setting off a chain reaction in which styrene molecules are added in a line.

Wolkow's work is some way from a commercial application, but if similar rows could be built from molecules that can transfer electrons, they could form the basis for wires, just one molecule wide, that would self-assemble on the surface of a premanufactured silicon chip.

\section{Attractive forces}

Others predict that standard manufacturing methods could be used to draw a circuit blueprint on the surface of a chip, and selfassembly techniques used to ferry prefabricated components to the appropriate places in that circuit. Angela Belcher of the University of Texas at Austin has recently identified a series of peptides - fragments of protein molecules - that attach themselves selectively to materials used in electronics, such as gallium arsenide ${ }^{5}$. Together with

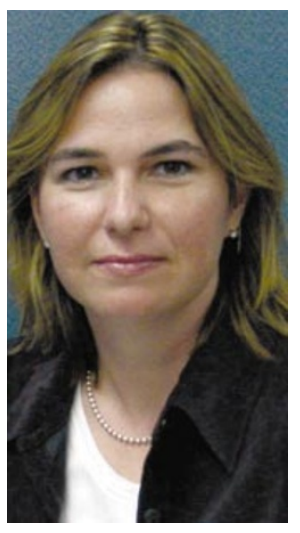

Angela Belcher hopes to self-assemble chips. division at IBM's Thomas J. Watson Research Center in Yorktown Heights, New York, thinks that the integration of self-assembly with conventional manufacturing techniques could have commercial applications. "We hope that self-assembly will be able to inexpensively replace certain stages in the production of materials and devices, where control is needed at the molecular level," he says.

The new commercial initiatives extend beyond microelectronics. Chad Mirkin and colleagues at Northwestern University in Evanston, Illinois, are exploiting the way in

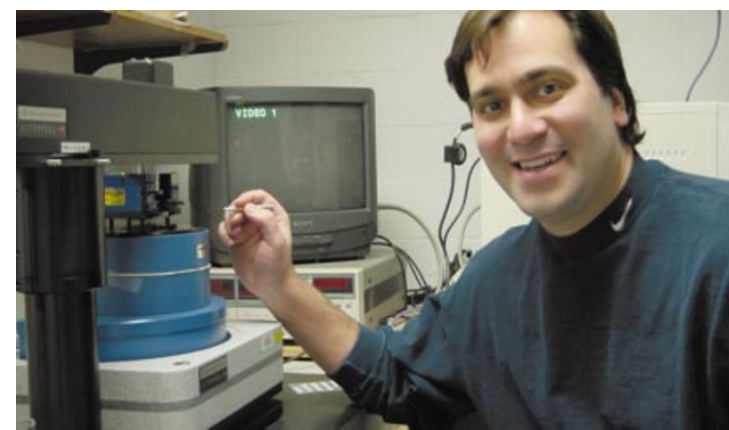

Gene screen: Chad Mirkin has launched a company to use self-assembly in genetic tests.

which DNA binds selectively to complementary sequences in the hope of developing a new technique for genetic screening ${ }^{6}$.

Mirkin first identifies the sequences of bases at either end of the DNA he hopes to screen for. He then creates two sets of strands of DNA, each complementary to one of the 'end' sequences of the target DNA. These are attached to gold nanoparticles, with each nanoparticle linked to just one type of strand, and the nanoparticles are dispersed in water.

If the target DNA is added to the solution, gold nanoparticles bind to both ends of the sequence using the complementary strands. The gold particles cluster together, eventually forming a large enough group to scatter light and change the colour of the solution. Last year Mirkin launched a company called Nanosphere, based in Northbrook, Illinois, to commercialize the idea.

Such emerging companies are evidence of the field's gradual move towards commercial applications. At the same time, the more blue-sky projects undertaken by Whitesides show that self-assembly has not lost its visionary zeal. Many in the field are still intent on creating the nanoscale chemical sensors or mechanical devices that are difficult or impossible to make by other methods. But success for the start-up companies should provide inspiration to all quarters of self-assembly research.

Fraser Stoddart, who works on selfassembling electronic devices at the University of California, Los Angeles, says that the field is moving a lot faster than he expected. "Something that I thought would only ever be a dream in my lifetime now stands a good chance of becoming a technological reality before this decade is out," he says.

\section{Phillip Ball is a consultant editor of Nature}

1. Clark, T. D., Tien, J., Duffy, D. C., Paul, K. E. \& Whitesides, G. M. J. Am. Chem. Soc. 123, 7677-7682 (2001).

2. Oliver, S. R. J., Clark, T. D., Bowden, N. \& Whitesides, G. M

J. Am Chem. Soc. 123, 8119-8120 (2001).

3. Gracias, D. H., Tien, J., Breen, T. L., Hsu, C. \& Whitesides, G. M. Science 289, 1170-1172 (2000).

4. Lopinski, G. P., Wayner, D. D. M. \& Wolkow, R. A. Nature 406, 48-51 (2000).

5. Whaley, S. R., English, D. S., Hu, E. L., Barbara, P. F. \& Belcher, A. M. Nature 405, 665-668 (2000).

6. Mirkin, C. A., Letsinger, R. L., Mucic, R. C. \& Storhoff, J. J. Nature 382, 607-609 (1996). 